\title{
Drying Characteristics and Quality of Yam Slices of Different Thickness during Microwave Intermittent Drying
}

\author{
Jian-Wu DAI ${ }^{1, a}$, Wen QIN ${ }^{2, \text { b }}$, Zhi-Jun WU ${ }^{1, \text { a }}$, Pai ZHANG ${ }^{1, \text { a }}$ and Li-Hua \\ ZHANG ${ }^{1, a}$ \\ ${ }^{1}$ College of Mechanical and Electrical Engineering, Sichuan Agricultural University, 625014, Ya'An, \\ Sichuan Province, China \\ ${ }^{2}$ College of Food Science, Sichuan Agricultural University, 625014 \\ Ya'An, Sichuan Province, China

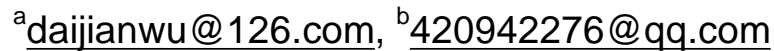

Keywords: yam slices, microwave intermittent drying, drying characteristic, Weibull model, quality Abstract. Homemade batch microwave drying test apparatus using different parameters on fresh-cut alpine yam slices during microwave intermittent drying experiments, the drying characteristics of fresh-cut alpine yam slices, the dynamic model and the drying quality was studied in the paper. The results showed that the whole microwave intermittent drying process of alpine yam slices generally includes acceleration, constant speed and deceleration stages. The slice thickness have a significant impact on shrinkage characteristics, and improve slice thickness had good influence on keeping the dry volume of samples.

\section{Introduction}

Wild yam grows in the high mountain area and has many species. Dopamine in yam can enhance body constitution, improve blood circulation and speed up the metabolism of skin, which make the skin more moist and bright color. Yam is rich in choline and lecithin to enhance memory. Fresh high mountain yam has a high moisture content approximately $76 \%$ (wet basis), which favors the growth of microorganisms [1-3]. Furthermore, the high moisture content can easily enhance enzymatic and non-enzymatic reactions, resulting in rapid deterioration of high mountain yam quality and thus lead to a reduction in the possible medicinal and commercial value. Therefore, employing suitable drying technology and appropriate drying conditions are of great practical importance. At present, the common processing methods are processed into pieces, powder, bionic food processing, yogurt processing, of which drying is one of the most important processes [4-6]. Microwave intermittent drying is a new drying technology developed in recent years. Compared with traditional drying methods, it can limit the temperature caused by food hardening, crusts and coking and even burn phenomenon. The utility model has the advantages of high utilization rate, short drying time, low energy cost, etc [7-12].

However, to date the literature from references and web resources, there is little information available about the characteristics of yam microwave intermittent drying. Guang-Yue Ren [13] studied the drying technology of yam by microwave drying. Meanwhile, the optimum process conditions were determined by 3 factors: energy consumption efficiency, drying rate and polysaccharide yield. Li Li [14] use drying temperature and slice thickness as the factors to study the heat pump drying characteristics of yam slices. In the meantime, SAS8.0 software was used to fit the experimental data. The heat pump drying model, drying rate curve and drying characteristic curve of heat pump drying were obtained. Xiao-Meng Ye [15] investigated vacuum microwave drying combined with freeze- drying instead of single freeze-drying to know the effects on the physical properties, sensory evaluation and ultra-structure of yam and reveal the characteristics of moisture migration during drying process. The objective of the current work was to investigate the drying kinetics of wild yam during microwave intermittent drying technology. Combined with its technical advantages, it can provide technical support and reference for the future industrialization of yam. 


\section{Materials and methods}

\section{Raw material}

Good-quality fresh yam tubers used in the present study were purchased from a local market in Ya'an, Sichuan Agricultural University. The samples were checked carefully to discard spoiled yam in order to prevent contamination by bacteria. To ensure the uniformity of the physical characteristics of experimental materials, the yam samples were selected with the same size. According to the State Administration of Medicine drug storage character number seventeenth document [16], the safe storage of water for the yam is $12 \%-17 \%$ (wet basis).

\section{Experimental setup and procedure}

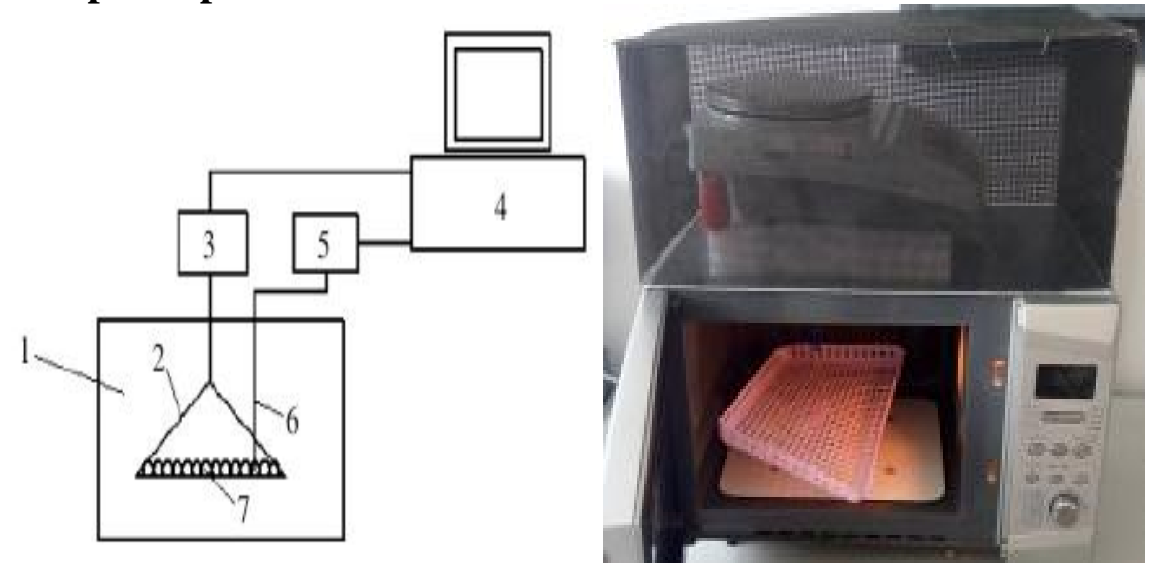

Fig. 1, The structure of microwave intermittent drying test device

Microwave intermittent drying test device as shown in Fig. 1. Microwave intermittent dryer was an experimental platform built on the basis of microwave oven. Continuous emission of microwaves can make fresh agricultural products or fruit surfaces drying .So it was the primary task to carry out intermittent control of microwave experiments. The whole drying process to the weight of the material had a real-time grasp, which was the weight of the material or the dehydration of the material over time with a real-time response. Therefore, a hole was drilled on the basis of the microwave oven. Then the material tray was lifted by the balance. Due to the discharge reaction, the material tray and wire cannot be metal. Now, in this paper, cotton and plastic tray were used as the modified material. The tray was hung on the hook of the balance by cotton thread. And the weight of the balance was read in real time to reflect a change in the state of material dehydration. As a hole in the microwave oven, microwave leakage may occur. And this kind of discharge reaction can be avoided by wire screen. In order to play the role of shielding, a very tight wire net was stuck on the hole.

The preliminary experimental results showed that the power of batch microwave drying test from high to low was $700 \mathrm{~W}, 600 \mathrm{~W}, 450 \mathrm{~W}$ and $250 \mathrm{~W}$, respectively. Fresh-cut yam slices were divided into four groups. A set of trials contained 4 levels, with four factors at four levels. The influence factors of yam slices thickness tests were studied. The above factors were used as variables to study the drying characteristics of yam slices in different conditions and to analyze the specific influence of each factor and level. Meanwhile, based on the results of preliminary experiment, the microwave power level was set at $700 \mathrm{~W}$ and the heating/intermittent time at $7 \mathrm{~s} / 240 \mathrm{~s}$, respectively. After each $30 \mathrm{~min}$, then weigh and recorded the test data during the experiment. And observe the quality changes of the sample in the experiment. When the yam slices drying to moisture content was less than $15 \%$, the experiment was ended.

Table 1, Design for experiments with drying parameters included

\begin{tabular}{ccccc}
\hline No. & $\begin{array}{c}\text { Slice thickness } \\
(\mathrm{mm})\end{array}$ & $\begin{array}{c}\text { Microwave } \\
\text { power }(\mathrm{W})\end{array}$ & $\begin{array}{c}\text { Heating } \\
\text { time }(\mathrm{s})\end{array}$ & $\begin{array}{c}\text { Intermittent } \\
\text { time }(\mathrm{s})\end{array}$ \\
\hline 1 & 2 & 700 & 7 & 240 \\
2 & 4 & 700 & 7 & 240 \\
3 & 6 & 700 & 7 & 240 \\
4 & 8 & 700 & 7 & 240 \\
\hline
\end{tabular}




\section{Calculation of moisture effective diffusivity}

The moisture ratio $(M R)$ of yam samples during drying experiments was calculated using Eq. 1: [17]

$$
M R=\frac{M_{t}}{M_{o}}
$$

Where $M_{0}$ is the initial dry base moisture content of wild yam, $M_{t}$ is the moisture content of drying base at any time.

The drying rate of yam samples during drying experiments were computed using Eq. 2: [18]

$$
D R=\frac{M_{t_{1}}-M_{t_{2}}}{t_{2}-t_{1}}
$$

Where $D R$ is the drying rate, $\% / \mathrm{h} ; \mathrm{M}_{t 1}$ is the moisture content of drying base at $\mathrm{t}_{1}, \mathrm{~g} / \mathrm{g} ; M_{t 2}$ is the moisture content of drying base at $t_{2}, \mathrm{~g} / \mathrm{g}$.

The calculation of moisture effective diffusivity is calculated using Eq. 3: [19, 20]

$$
\ln (M R)=\ln \left(\frac{6}{\pi^{2}}\right)-\left(\pi^{2} \frac{D_{\text {eff }} t}{r_{o}{ }^{2}}\right)
$$

There is a linear relationship between the natural logarithm of moisture ratio and drying time in the wild yam according to Eq. 3. While the slope is $-\pi^{2} D_{e f f} / H^{2}$, drying moisture ratio $M R$ and drying time $\mathrm{t}$ are known quantities, then the slope is obtained by linear regression. Put k into the formula Eq. 3 and moisture effective diffusivity gets clearly.

\section{Evaluation method of shrinkage characteristics of wild yam}

An important index to evaluate the quality after drying is shrinkage characteristic, which is directly related to the taste, appearance quality and rehydration characteristics of fruits and vegetables. Regarding it as flat slab according to it's material characteristics. The volume change mainly along with the thickness direction. Therefore, the measured thickness of three different representative positions can be compared among the dried yam.

\section{Results and discussion}

Effect of different slice thickness on drying characteristics of wild yam

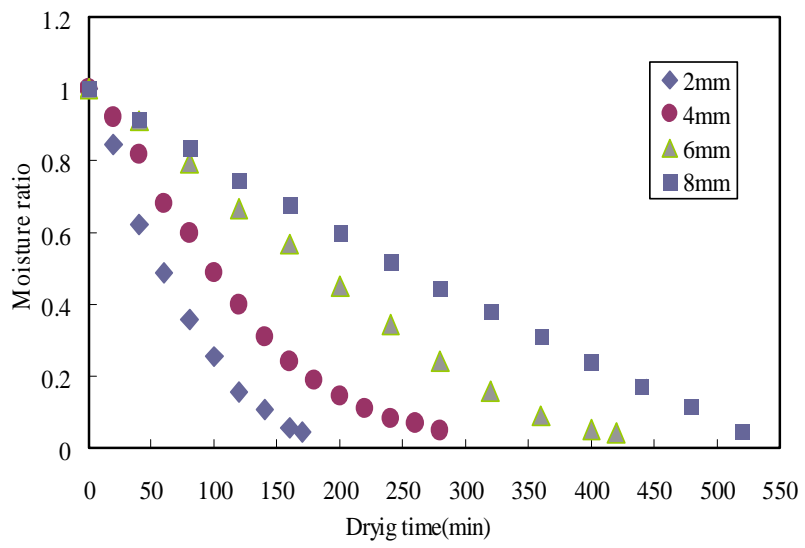

(a)

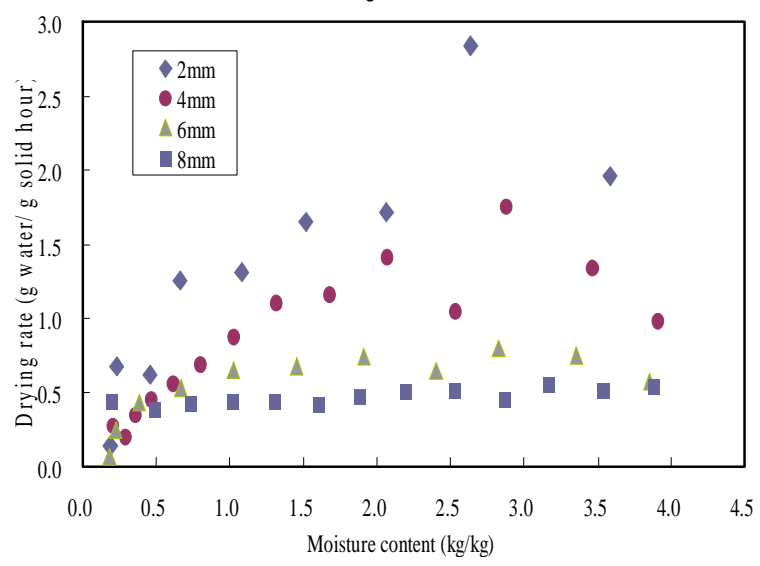

(b)

Fig. 2, Drying kinetics of yam slices at different sample slice thickness with the heating/intermittent time of $7 / 240$ s and the microwave power of $700 \mathrm{~W}$

The fig. 2a showed the slice thickness had great influence on the drying curve of mountain wild yam slices. That was, the greater the microwave intensity and the faster the drying rate, the shorter of the entire drying time required to the safe moisture content. At the end of drying stages, the curve became gentle and the difficulty of microwave drying significantly increased. It can be seen that there was a positive correlation between drying rate and microwave power.

The fig. $2 \mathrm{~b}$ showed in the same case with different slice thickness, the drying rate contained obvious distinction. Holding any other factors constant, the greater the slice thickness, the slower the drying 
rate. This was because when the material was thinner, the shorter the distance between the internal moisture migration. For a certain volume of material, as the surface area became thicker, the relative surface area in contact with air would become smaller. In the meantime, the great internal heat transfer resistance affected heat and mass transfer. So the drying rate was much slower. The drying process of $2 \mathrm{~mm}$ and $4 \mathrm{~mm}$ didn't contained constant stage from the drying curve. While it contained three drying stages in $6 \mathrm{~mm}$ drying process, including the beginning of the speed stage, constant speed stage, and the final deceleration stage. The constant speed stage had a long duration. The drying process was in a constant speed stage at the thickness level of $8 \mathrm{~mm}$.

\section{Moisture effective diffusivity under different slice thickness}

Under different slice thickness, moisture diffusivities of alpine wild yams in drying process were as follows in the Table 2.

Table 2, Moisture effective diffusion coefficients under different slice thickness

\begin{tabular}{cccc}
$\begin{array}{c}\text { Slice thickness } \\
(\mathrm{mm})\end{array}$ & $\begin{array}{c}\text { Linear regression fitting } \\
\text { formula }\end{array}$ & $\mathrm{R}^{2}$ & $\mathrm{D}_{\text {eff }}\left(10^{-9} \mathrm{~m}^{2} / \mathrm{s}\right)$ \\
\hline 2 & $\operatorname{lnMR}=-0.3108 \mathrm{t}+0.2686$ & 0.9724 & 1.26 \\
4 & $\operatorname{lnMR}=-0.1854 \mathrm{t}+0.2625$ & 0.9846 & 3.00 \\
6 & $\operatorname{lnMR}=-0.1270 \mathrm{t}+0.4164$ & 0.9363 & 4.63 \\
8 & $\operatorname{lnMR}=-0.0832 \mathrm{t}+0.3308$ & 0.8774 & 5.40 \\
\hline
\end{tabular}

As we can see from the form, the $D_{\text {eff }}$ values of samples at different thickness for the microwave power with $700 \mathrm{~W}$ changed from $1.26 \times 10^{-9}$ to $5.40 \times 10^{-9} \mathrm{~m}^{2} / \mathrm{s}$. When the slice thickness was $8 \mathrm{~mm}$, the moisture effective diffusion coefficient was 4.29 times higher than that of $2 \mathrm{~mm}$ during the process, which indicated that increasing slice thickness can improve the moisture effective diffusion rate of the yam. For microwave intermittent drying of wild yam, slice thickness had a significant effect on the moisture effective diffusion coefficient. To some extent, increasing the slice thickness can improve the efficiency of moisture effective diffusion. This was just contrary to the general law. The reason was that the high thickness was beneficial to maintain the temperature inside the material. The thinner the thickness was, the easier it was to cool down, which was not conducive to keep the temperature.

\section{Effects of different factors on the contraction of the alpine yam slices}

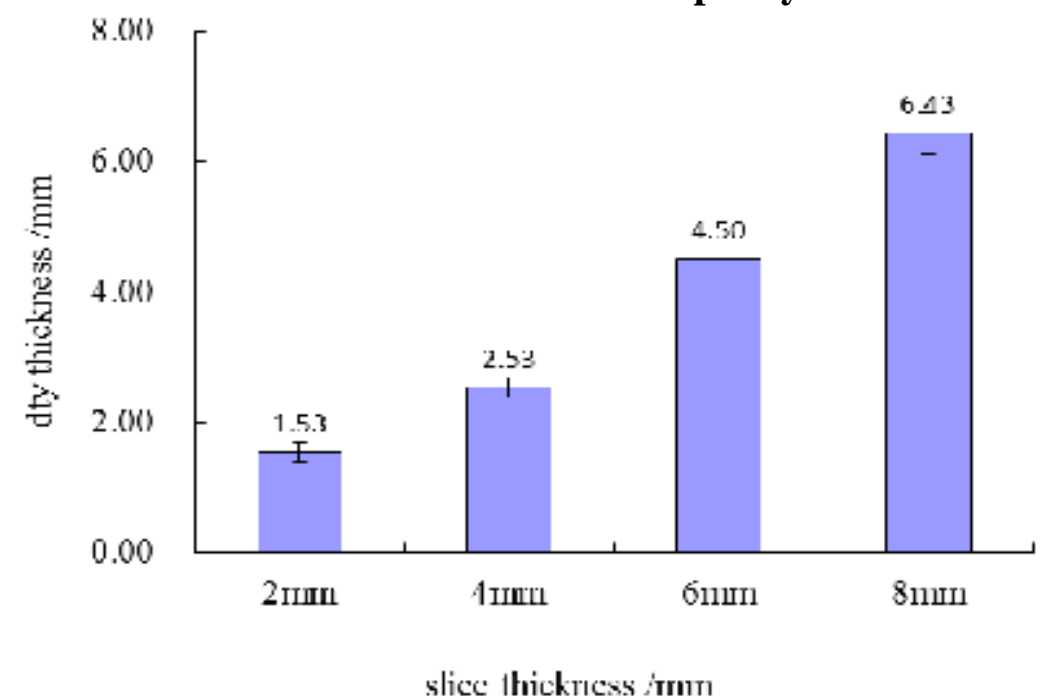

Fig. 3, Drying contraction of yam slices at different thickness

Fig.3 illustrated that with the increase of slice thickness, the dry thickness of the yams showed a processing trend. The contraction percentage varied from $19.6 \%$ to $36.8 \%$ when the thickness increasing from $2 \mathrm{~mm}$ to $8 \mathrm{~mm}$. The results indicated that increasing the thickness of material can be conducive to keep good quality in terms of dried volume. 


\section{Conclusions}

(1) The study showed that slice thickness had great effect on drying time. The drying time decreased with the decrease of slice thickness. The drying rate curve of the four levels shows that alpine yam slices during microwave intermittent drying process generally includes acceleration, constant speed and deceleration phases. However, the constant speed stage was inconspicuous in the curve.

(2) When slice thickness changed from $2 \mathrm{~mm}$ to $8 \mathrm{~mm}$ with the power of $700 \mathrm{~W}$, the moisture effective diffusivity varied from $1.26 \times 10^{-9}$ to $5.40 \times 10^{-9} \mathrm{~m}^{2} / \mathrm{s}$, and the contraction percentage varied from $19.6 \%$ to $36.8 \%$. Increasing slice thickness can improve the moisture effective diffusion rate of the yam, and it also can be conducive to keep good quality in terms of dried volume.

\section{Acknowledgements}

The authors gratefully acknowledge the Natural Science Project of Sichuan (No.035Z1986) for providing financial support to this investigation.

\section{References}

[1] Ming Jiang, Mao-Yang Rao. Research on the processing of dehydrated yam slices[J]. Anhui Agricultural Sciences, 2006, 34(16): 4095-4100.

[2] Cheng-He Zhou, Yun Wu, Qinchang Liu. Processing and utilization of Chinese yam[J]. Bulletin of Anhui Agricultural Sciences, 2014, 10 (4): 65-66.

[3] Jin-Da Hao, Zong-Wan Xie. On the new medicinal materials in history[J]. Chinese Journal of Traditional Chinese Medicine, 2011, 19(11): 643-645.

[4] Bing Bai, Ming-Jing Li, Juan Zhao. Study on the constituents of Chinese yam[J]. Chinese Journal of Traditional Chinese Medicine, 2015, 33(11): 1271-1274.

[5] Jun Zhao, An-Jian Wang, Xue-Wei Fan. Microwave vacuum freeze-drying of yam chip preparation[J]. Food Science and Technology, 2016, 5(27): 89-91.

[6] Chong-Wen Cao. Current status of microwave vacuum drying[J]. Drying Technology and Equipment, 2014, 2(3): 5-9.

[7] Zhi-Jun Liu. Study on microwave drying characteristics of wood[D]. Beijing Forestry University, 2016.

[8] Li-Hua Zhang, Yan Zheng, Wen Qin. Optimization of vacuum microwave drying parameters of Chinese prickly ash[J]. Journal of Southwestern University (NATURAL SCIENCE EDITION), 2008, 30(7): 179-183.

[9] Li-Hua Zhang, Wen Zhang. Optimization of lees intermittent microwave drying technology of agricultural engineering[J]. Journal of Response Surface Method, 2011, 27(3): 369-374.

[10] Jun Wang, Yong-Sen Xiong. Microwave drying characteristics of potato and the effect of different microwave powers on the dried quality of potato[J].European Food Research and Technology, 2014, 5(219): 500-506.

[11] Duygu Evin. Microwave drying and moisture diffusivity of white mulberry: experimental and mathematical modeling[J]. Journal of Mechanical Science and Technology, 2011, 10(25): 2711-2718.

[12] B. E. Prasad, Krishna K. Pandey. Microwave drying of bamboo[J]. European Journal of Wood and Wood Products, 2012, 3(70): 353-355.

[13] Guang-Yue Ren. Microwave drying characteristics of Chinese yam[J]. Food Science, 2010, 31 (22): 203-206. 
[14] Li Li, Ji-An Sun, Jin-Feng Sheng. Pump drying characteristics and mathematical model of yam[J]. Modern Food Science and Technology, 2014, 30(10): 212-217.

[15] Xiao-Meng Ye. Freeze-dried of combined drying process of microwave vacuum drying[D]. Shihezi University, 2014.

[16] Yan-Zhen Chen. Study on microwave vacuum drying of Chinese yam[D]. Henan University of Science and Technology, 2009.

[17] Yuan-Yuan Chen, Yun-Peng Fu, Zhi-Jun Wang. Microwave vacuum drying treatment yam polysaccharide effect rate and drying characteristics[J]. Processing of Agricultural Products, 2012, 11(4): 99-102.

[18] Yan-Bin Huang, You Zheng. Hot air drying characteristics and mathematical model of lemon[J]. Food industry science and technology, 2012, 33(14): 169-191.

[19] Chao Zhao. Experimental study on intermittent microwave drying theory and process optimization of Chinese prickly ash[D]. Beijing, Southwestern University, 2012.

[20] Chang-Long Li. Study on the characteristics and new technology of microwave heat pump combined drying ammonium sulfate[D]. Kunming University of Science and Technology, 2010. 